\title{
Dynamics Analysis of a Delayed Rumor Propagation Model in an Emergency-Affected Environment
}

\author{
Chunru $\mathrm{Li}^{1,2}$ and Zujun $\mathrm{Ma}^{3}$ \\ ${ }^{1}$ School of Transportation and Logistics, Southwest Jiaotong University, Chengdu 610031, China \\ ${ }^{2}$ Huaian College of Information Technology, Huaian 223003, China \\ ${ }^{3}$ School of Economics and Management, Southwest Jiaotong University, Chengdu 610031, China \\ Correspondence should be addressed to Chunru Li; lichunru88@126.com
}

Received 11 May 2015; Revised 15 July 2015; Accepted 28 July 2015

Academic Editor: Luca Gori

Copyright (C) 2015 C. Li and Z. Ma. This is an open access article distributed under the Creative Commons Attribution License, which permits unrestricted use, distribution, and reproduction in any medium, provided the original work is properly cited.

\begin{abstract}
Rumors influence people's decisions in an emergency-affected environment. How to describe the spreading mechanism is significant. In this paper, we propose a delayed rumor propagation model in emergencies. By taking the delay as the bifurcation parameter, the local stability of the boundary equilibrium point and the positive equilibrium point is investigated and the conditions of Hopf bifurcation are obtained. Furthermore, formulas for determining the direction of Hopf bifurcation and the stability of the bifurcating periodic solutions are derived by applying the normal form method and center manifold theorem. Finally, some numerical simulations are also given to illustrate our theoretical results.
\end{abstract}

\section{Introduction}

Emergencies usually cause serious negative impacts on people's work and life in a variety of ways. For example, the event itself may lead to financial loss or personal injuries, and also at the same time the rumor may begin to prevail and it leads to panic feelings or irrational behavior. Rumor is usually defined as the unconfirmed elaboration or annotation of the public interesting things, events, or issues that spread through various channels [1-3]. When emergencies occur, people often fail to reflect rumor's seriousness and take active measures to deal with the related issues in time. Rumor especially the exaggerated rumor, as a product of emergencies, may bring helplessness and distress to the public, hamper the regulation of public opinions, and simultaneously destroy social harmony and stability. For instance, during the 2011 earthquake and Fukushima nuclear leak in Japan, because of the propagation of the rumors about the water pollution, many people in China blindly rush to buy salts, which greatly affects the regular social order [4]. Thus, how to effectively control and prevent rumor propagation in emergencies has become one of the most valuable research topics.
Accurate analysis of the dynamic behavior of rumor propagation is significant for controlling and preventing rumor propagation in emergencies. Using mathematical models to analyze the dynamic characteristics about rumor propagation has now become a compelling topic [5-9]. Rumor propagation is very similar to the diffusion of the virus; thus, most of the existing models of rumor propagation are derived from the models of infectious diseases. The classical models (i.e., the DK model) for rumor propagation were introduced by Daley and Kendall [10]. They mainly divided people into the following three classes: ignorants (those who cannot identify the rumors), spreaders (those who spread rumors), and stiflers (those who do not believe the rumors again). Thenceforth, most of the researchers applied or improved DK model to study rumor propagation. Maki and Thompson applied a mathematical model to study rumor propagation, and they mainly focused on the theoretical analysis [11]. Z.-L. Zhang and Z.-Q. Zhang [12] supposed that the emergency state and the spreading rate of rumors were continuous functions of time. Then, they established a rumor propagation model in emergencies based on three-molecule models. Their results showed that rumor spreading at certain rate had positive effects on situation 
stability and especially had delaying effects on the rapid proliferation of an emergency. Based on the analysis of [12], Zhao [13] considered the impact of authorities' media and rumor propagation on the evolution of emergencies, and they presented a new rumor propagation model with the impact of authorities' media. Huo [14] studied a rumor transmission model with constant immigration and incubation. Their analysis results meet the actual circumstances better and more externally, and they are easier to understand. Zhao and Wang [15], based on epidemic-like models, established a ISRW dynamical model considering the medium to describe the rumor propagation in emergencies. They discussed the stability of the rumor propagation model and suggested that the government should enhance the management of Internet. Recently, Zhang et al. [16] considered an 8-state ICSAR rumor propagation model with official rumor refutation. They believed that high spread probability and low block value could lead to rumor refutation. However, this model did not consider the impact of delays on rumor propagation.

In fact, delays always exist in rumor propagation. For example, when rumor susceptible people are infected by rumor spreading people, there is a time during which the infected people can have the ability to spread rumor. On the other hand, when rumor spreading people are educated by our government, there also exists a delayed time after which the rumor spreading people can really recover and will not believe the rumor. Therefore, delay is an important factor in rumor propagation.

Inspired by the abovementioned work, this paper deals with a novel delayed rumor propagation model with media report, which is of the following form:

$$
\begin{aligned}
& \frac{d S}{d t}=A\left(1-\frac{S}{S+m}\right)-\beta S I-\mu S, \\
& \frac{d I}{d t}=\beta S I-\mu I-\alpha I(t-\tau), \\
& \frac{d R}{d t}=\alpha I(t-\tau)-\mu R
\end{aligned}
$$

with initial conditions

$$
\begin{gathered}
S(t)=S_{0}, \quad t \in[-\tau, 0], \\
I(t)=I_{0}, \quad t \in[-\tau, 0], \\
R(t)=R_{0}, \quad t \in[-\tau, 0],
\end{gathered}
$$

where $S(t)$ is the density of the rumor susceptible people at time $t, I(t)$ is the density of the rumor infected people at time $t, R(t)$ is the density of the rumor recovered people at time $t$, and the coefficients $A, m, \alpha, \beta, \mu$ are positive constants. $A$ represents the natural growth rate of the rumor susceptible people, $m$ represents the impact of media report, $\alpha$ represents the impact of our government on the rumor infected people, $\beta$ is the infected rate, and $\mu$ describes a rate that people remove from the system since they lose interest in rumors [17]. $\tau \geq 0$ is a delay, which implies that when rumor infected people are educated by our government, there exists a delayed time after which the rumor spreading people can really recover. $S_{0}, I_{0}, R_{0}$ are the initial densities of the rumor susceptible people, the rumor infected people, and the rumor recovered people, respectively.

Accordingly, the remaining structure of this paper is arranged as follows. In Section 2, we are concerned with local stability and Hopf bifurcation of the equilibrium points for our model. In Section 3, the properties of Hopf bifurcation, formula determining the direction of Hopf bifurcation, and the stability of spatially homogeneous bifurcating periodic solutions are investigated. In Section 4, numerical simulations are presented to demonstrate our theoretical results. Finally, conclusions are drawn in Section 5.

\section{Local Stability and Hopf Bifurcation}

Because the first two equations in (1) are independent of $R(t)$, we can consider the following reduced model:

$$
\begin{aligned}
& \frac{d S}{d t}=A\left(1-\frac{S}{S+m}\right)-\beta S I-\mu S, \\
& \frac{d I}{d t}=\beta S I-\mu I-\alpha I(t-\tau),
\end{aligned}
$$

with initial conditions

$$
\begin{array}{ll}
S(t)=S_{0}, & t \in[-\tau, 0], \\
I(t)=I_{0}, & t \in[-\tau, 0] .
\end{array}
$$

Obviously, system (3) has the following two kinds of equilibria points:

(i) The boundary equilibrium point $E^{1}=((-m \mu+$ $\sqrt{m \mu(m \mu+4 A)}) / 2 \mu, 0)^{T}$.

(iii) If $\left(H_{1}\right) A m \beta^{2}-\mu(\mu+\alpha)(m \beta+\alpha+\mu)>0$ holds, then there exists the positive equilibrium point

$$
E^{*}=\left(\frac{\alpha+\mu}{\beta}, \frac{m A \beta^{2}-\mu(\mu+\alpha)(m \beta+\alpha+\mu)}{\beta(\mu+\alpha)(m \beta+\alpha+\mu)}\right)^{T} \text {. }
$$

In this part, we will discuss the local stability and Hopf bifurcation of the boundary equilibrium point $E^{1}$ and the positive equilibrium point $E^{*}$ with the time delay $\tau$ as the bifurcation parameter.

For further discussion, we firstly make the following assumptions:

$$
\begin{aligned}
& \left(H_{2}\right) \beta[-m \mu+\sqrt{m \mu(m \mu+4 A)}]-2 \mu(\mu+\alpha)<0, \\
& \left(H_{3}\right) \beta[-m \mu+\sqrt{m \mu(m \mu+4 A)}+2 \mu(\alpha-\mu)]>0, \\
& \left(H_{4}\right)(\mu-\alpha)(\beta m+\alpha+\mu)-2 \alpha(\alpha+\mu)<0 .
\end{aligned}
$$

2.1. Stability and Hopf Bifurcation of the Boundary Equilibrium Point $E^{1}$. By a simple calculation, it is easy to obtain the characteristic equation of system (3) at the boundary equilibrium point $E^{1}$ as the following form:

$$
\left[\lambda+\mu+\frac{m}{\left(m+S_{0}\right)^{2}}\right]\left(\lambda-\beta S_{0}+\mu+\alpha e^{-\lambda \tau}\right)=0,
$$

where $S_{0}=(-m \mu+\sqrt{m \mu(m \mu+4 A)}) / 2 \mu$. 
Lemma 1. If $\left(\mathrm{H}_{2}\right)$ holds, then the boundary equilibrium point $E^{1}$ of system (3) with $\tau=0$ is locally asymptotically stable.

Proof. Clearly, (6) always has a negative real root; namely,

$$
\lambda=-\mu-\frac{m}{\left(m+S_{0}\right)^{2}} .
$$

Therefore, we only consider the form of

$$
\lambda-\beta S_{0}+\mu+\alpha e^{-\lambda \tau}=0 .
$$

When $\tau=0$, (8) can be rewritten as

$$
\lambda-\beta S_{0}+\mu+\alpha=0
$$

Obviously, under condition $\left(H_{2}\right)$,

$$
\begin{aligned}
\lambda & =\frac{1}{2 \mu}\{\beta[-m \mu+\sqrt{m \mu(m \mu+4 A)}]-2 \mu(\mu+\alpha)\} \\
& <0 .
\end{aligned}
$$

Thus, the boundary equilibrium point $E^{1}$ is locally asymptotically stable as $\tau=0$.

Now we discuss the effect of the delay $\tau$ on the stability of $E^{1}$. Assume that $i \omega$ is a root of (8). Then $\omega$ should satisfy the following equations:

$$
\begin{aligned}
& \alpha \cos \omega \tau=\beta S_{0}-\mu, \\
& \alpha \sin \omega \tau=\omega .
\end{aligned}
$$

Taking square on both sides of the equations of (11) and summing them up, we obtain

$$
\omega^{2}=\alpha^{2}-\left(\beta S_{0}-\mu\right)^{2}
$$

If $\left(\mathrm{H}_{2}\right)$ and $\left(\mathrm{H}_{3}\right)$ hold, then (12) has a positive real root $\omega_{+}$, and

$$
\omega_{+}=\sqrt{\alpha^{2}-\left(\beta S_{0}-\mu\right)^{2}} .
$$

Therefore, according to (11), it is easy to show that

$$
\tau_{i}=\frac{1}{\omega}\left(\arccos \frac{S_{0} \beta-\mu}{\alpha}+2 i \pi\right) \quad(i=0,1,2, \ldots) .
$$

Furthermore, differentiating both sides of (8) with respect to $\tau$, we have

$$
\frac{d \lambda}{d \tau}=\frac{1}{\alpha^{2}}>0
$$

Based on the above analysis, we obtain the following result.

Theorem 2. If $\left(\mathrm{H}_{2}\right) \sim\left(\mathrm{H}_{3}\right)$ hold, then the boundary equilibrium point $E^{1}$ of system (3) is locally asymptotically stable when $\tau \in$ $\left[0, \tau_{0}\right)$. Moreover, it undergoes a Hopf bifurcation when $\tau=\tau_{i}$.
2.2. Stability and Hopf Bifurcation of the Positive Equilibrium Point $E^{*}$. For the positive equilibrium point $E^{*}$, the characteristic equation of system (3) is as follows

$$
\begin{aligned}
\lambda^{2} & +\left[\frac{A m \beta^{2}}{(\mu+\alpha)(m \beta+\alpha+\mu)}-\alpha+\frac{A m \beta^{2}}{(m \beta+\alpha+\mu)^{2}}\right] \lambda \\
& +\left[\frac{A m \mu \beta^{2}}{(\mu+\alpha)(m \beta+\alpha+\mu)}-\frac{A m \alpha \beta^{2}}{(m \beta+\alpha+\mu)^{2}}\right. \\
& -\mu(\mu+\alpha)]+\left[\lambda+\frac{A m \beta^{2}}{(\mu+\alpha)(m \beta+\alpha+\mu)}\right. \\
& \left.+\frac{A m \beta^{2}}{(m \beta+\alpha+\mu)^{2}}\right] \alpha e^{-\lambda \tau}=0 .
\end{aligned}
$$

Lemma 3. If $\left(\mathrm{H}_{1}\right)$ holds, then the positive equilibrium point $E^{*}$ of system (3) with $\tau=0$ is locally asymptotically stable.

Proof. When $\tau=0$, we have

$$
\begin{aligned}
\lambda^{2}+ & \frac{A m \beta^{2}}{m \beta+\alpha+\mu}\left(\frac{1}{\mu+\alpha}+\frac{1}{\beta m+\alpha+\mu}\right) \lambda \\
& +\frac{1}{\beta m+\alpha+\mu}\left[A m \beta^{2}-\mu(\mu+\alpha)(m \beta+\alpha+\mu)\right] \\
& =0 .
\end{aligned}
$$

Under condition $\left(H_{1}\right)$, it is easy to obtain

$$
A m \beta^{2}-\mu(\mu+\alpha)(m \beta+\alpha+\mu)>0 .
$$

According to the Routh-Hurwitz criteria, all the roots of (17) have negative real parts. Therefore, when $\tau=0$, the positive equilibrium point $E^{*}$ is locally asymptotically stable.

Next we discuss the effect of the delay $\tau$ on the stability of $E^{*}$. Assume that $i \omega(\omega>0)$ is a root of (16). Then $\omega$ should satisfy the following equation:

$$
\begin{gathered}
\alpha\left\{\left[\frac{A m \beta^{2}}{(\mu+\alpha)(\beta m+\alpha+\mu)}+\frac{A m \beta^{2}}{(\beta m+\alpha+\mu)^{2}}\right] \cos \omega \tau\right. \\
+\omega \sin \omega \tau\}=\omega^{2}-\left[\frac{A m \mu \beta^{2}}{(\mu+\alpha)(m \beta+\alpha+\mu)}\right. \\
\left.-\frac{A m \alpha \beta^{2}}{(m \beta+\alpha+\mu)^{2}}-\mu(\mu+\alpha)\right], \\
\alpha\left\{\left[-\frac{A m \beta^{2}}{(\mu+\alpha)(\beta m+\alpha+\mu)}+\frac{A m \beta^{2}}{(\beta m+\alpha+\mu)^{2}}\right]\right.
\end{gathered}
$$




$$
\begin{aligned}
& \cdot \sin \omega \tau+\omega \cos \omega \tau\} \\
& =-\omega\left[\frac{A m \beta^{2}}{(\mu+\alpha)(m \beta+\alpha+\mu)}-\alpha\right. \\
& \left.+\frac{A m \beta^{2}}{(m \beta+\alpha+\mu)^{2}}\right],
\end{aligned}
$$

which implies

$$
\begin{aligned}
& \omega^{4}+\omega^{2}\left[\left(A_{1}+A_{2}-\alpha\right)^{2}\right. \\
& \left.\quad-2\left(A_{1} \mu-A_{2} \alpha-\alpha \mu-\mu^{2}\right)-\alpha^{2}\right] \\
& +\left[\left(A_{1} \mu-A_{2} \alpha-\alpha \mu-\mu^{2}\right)^{2}-\alpha^{2}\left(A_{1}+A_{2}\right)^{2}\right] \\
& \quad=0
\end{aligned}
$$

where

$$
\begin{aligned}
& A_{1}=\frac{A m \beta^{2}}{(\alpha+\mu)(\beta m+\alpha+\mu)}, \\
& A_{2}=\frac{A m \beta^{2}}{(\beta m+\alpha+\mu)^{2}} .
\end{aligned}
$$

$$
\begin{aligned}
z_{0} & =\frac{1}{2}\left\{-\left(A_{1}+A_{2}\right)^{2}+2(\mu+\alpha)\left(A_{1}-\mu\right)\right. \\
& \left.+\sqrt{\left[\left(A_{1}+A_{2}\right)^{2}-2(\mu+\alpha)\left(A_{1}-\mu\right)\right]^{2}-4\left[\left(A_{1} \mu-A_{2} \alpha-\alpha \mu-\mu^{2}\right)^{2}-\alpha^{2}\left(A_{1}+A_{2}\right)^{2}\right]}\right\} .
\end{aligned}
$$

Furthermore, we obtain $\omega_{0}=\sqrt{z_{0}}$. This completes the proof.

Now, from (19), it is easy to show that

$$
\begin{aligned}
\tau_{j}^{*} & =\frac{1}{\omega_{0}}\left[\arccos \frac{\left(A_{1}+A_{2}\right)\left(-A_{1} \mu+A_{2} \alpha+\alpha \mu+\mu^{2}\right)+\alpha \omega_{0}^{2}}{\alpha\left[\left(A_{1}+A_{2}\right)^{2}+\omega_{0}^{2}\right]}\right. \\
& +2 j \pi] .
\end{aligned}
$$

Lemma 5 (see [18]). Let $\lambda(\tau)=\vartheta(\tau) \pm i \omega(\tau)$ be the root of (16) near $\tau=\tau_{0}^{*}$ satisfying $\vartheta\left(\tau_{0}^{*}\right)=0, \omega\left(\tau_{0}^{*}\right)=\omega_{0}$. Suppose that $h^{\prime}\left(\omega_{0}^{2}\right)>0$. Then $\pm i \omega_{0}$ is a pair of simple purely imaginary
Let $z=\omega^{2}$; then

$$
\begin{aligned}
& z^{2}+z\left[\left(A_{1}+A_{2}-\alpha\right)^{2}-2\left(A_{1} \mu-A_{2} \alpha-\alpha \mu-\mu^{2}\right)\right. \\
& \left.-\alpha^{2}\right]+\left[\left(A_{1} \mu-A_{2} \alpha-\alpha \mu-\mu^{2}\right)^{2}\right. \\
& \left.-\alpha^{2}\left(A_{1}+A_{2}\right)^{2}\right]=0 .
\end{aligned}
$$

Without loss of generality, define

$$
\begin{aligned}
& h(z)=z^{2}+z\left[\left(A_{1}+A_{2}-\alpha\right)^{2}\right. \\
& \left.\quad-2\left(A_{1} \mu-A_{2} \alpha-\alpha \mu-\mu^{2}\right)-\alpha^{2}\right] \\
& \quad+\left[\left(A_{1} \mu-A_{2} \alpha-\alpha \mu-\mu^{2}\right)^{2}-\alpha^{2}\left(A_{1}+A_{2}\right)^{2}\right] .
\end{aligned}
$$

Lemma 4. If $\left(\mathrm{H}_{4}\right)$ holds, then (20) has a unique positive real root.

Proof. Under condition $\left(H_{4}\right)$, a simple calculation shows that

$$
\begin{aligned}
& \left(A_{1} \mu-A_{2} \alpha-\alpha \mu-\mu^{2}\right)^{2}-\alpha^{2}\left(A_{1}+A_{2}\right)^{2} \\
& =\frac{1}{\beta m+\alpha+\mu}\left[A m \beta^{2}-\mu(\mu+\alpha)(m \beta+\alpha+\mu)\right] \\
& \quad \cdot\left\{\frac{A m \beta^{2}}{(\mu+\alpha)(m \beta+\mu+\alpha)^{2}}[(\mu-\alpha)(\beta m+\alpha+\mu)\right. \\
& -2 \alpha(\alpha+\mu)]-\mu(\mu+\alpha)\}<0 .
\end{aligned}
$$

Thus, (22) has a unique positive real root; namely,

roots of (16). Moreover, the following transversality condition holds:

$$
\left.\frac{d(\operatorname{Re} \lambda(\tau))}{d \tau}\right|_{\tau=\tau_{0}^{*}, \lambda=i \omega_{0}}>0 .
$$

Applying Lemma 3-Lemma 5, we have the following result.

Theorem 6. If $\left(H_{1}\right)$ and $\left(H_{4}\right)$ hold. Further suppose that $h^{\prime}\left(\omega_{0}^{2}\right)>0$ satisfies. Then

(i) the positive equilibrium point $E^{*}$ of system (3) is locally asymptotically stable when $\tau \in\left[0, \tau_{0}^{*}\right)$;

(ii) system (3) undergoes a Hopf bifurcation at $E^{*}$ when $\tau=$ $\tau_{0}^{*}$. 


\section{Properties of Hopf Bifurcation}

In this section, for the positive equilibrium point $E^{*}$ we will investigate the direction of spatially homogeneous Hopf bifurcation and the stability of bifurcated periodic solutions by using the theory of $[19,20]$.
Denote $\tau_{0}^{*}$ by $\tau^{*}$ and introduce the new parameter $\gamma=$ $\tau-\tau^{*}$. Normalizing the delay $\tau$ by the time scaling $t \rightarrow t / \tau$, then system (3) can be rewritten as

where for $\varphi=\left(\varphi_{1}, \varphi_{2}\right)^{T} \in \mathscr{C}$.

The linearized system of $(28)$ at $(0,0)$ is

$$
\frac{d U(t)}{d t}=L\left(\tau^{*}\right)\left(U_{t}\right)
$$

Based on the discussion in Section 2, we can easily know that for $\tau=\tau^{*}$ the characteristic equation of (30) has a pair of simple purely imaginary eigenvalues $\Lambda_{0}=\left\{i \omega_{0} \tau^{*},-i \omega_{0} \tau^{*}\right\}$.

Let $\mathscr{C}:=C\left([-1,0], \mathbb{R}^{2}\right)$. By the Riesz representation theorem, there exists a $2 \times 2$ matrix function $\eta(\theta, \tau),-1 \leq$ $\theta \leq 0$, whose elements are of bounded variation such that

$$
L\left(\tau^{*}\right)(\varphi)=\int_{-1}^{0}\left[d \eta\left(\theta, \tau^{*}\right)\right] \varphi(\theta), \quad \text { for } \varphi \in C .
$$

In fact, we can choose

$$
\begin{aligned}
\eta\left(\theta, \tau^{*}\right) & =\left(\begin{array}{cc}
-\frac{A m \beta^{2}}{\beta m+\mu+\alpha}\left(\frac{1}{\beta m+\mu+\alpha}+\frac{1}{\mu+\alpha}\right)-(\alpha+\mu) \\
\left(\frac{A m \beta^{2}}{(\beta m+\mu+\alpha)(\mu+\alpha)}-\mu\right) & \alpha
\end{array}\right) \delta(\theta) \\
& -\tau^{*}\left(\begin{array}{cc}
0 & 0 \\
0 & -\alpha
\end{array}\right) \delta(\theta+1),
\end{aligned}
$$

where $\delta(0)=1, \delta(\theta)=0(-1 \leq \theta<0)$.
Let $A\left(\tau^{*}\right)$ denote the infinitesimal generator of the semigroup induced by the solutions of (30) and let $A^{*}$ be the formal adjoint of $A\left(\tau^{*}\right)$ under the bilinear pairing

$$
\begin{aligned}
(\psi, \phi)= & (\psi(0), \phi(0)) \\
& -\int_{-1}^{0} \int_{\xi=0}^{\theta} \psi(\xi-\theta) d \eta(\theta) \phi(\xi) d \xi \\
= & (\psi(0), \phi(0)) \\
& +\tau^{*} \int_{-1}^{0} \psi(\theta+1)\left(\begin{array}{cc}
0 & 0 \\
0 & -\alpha
\end{array}\right) \phi(\theta) d \theta,
\end{aligned}
$$

for $\phi \in C, \psi \in C^{*}=C\left([0,1], R^{2}\right)$. Then $A\left(\tau^{*}\right)$ and $A^{*}$ are a pair of adjoint operators. From the discussion in Section 2, we know that $A\left(\tau^{*}\right)$ has a pair of simple purely imaginary eigenvalues $\pm i \omega_{0} \tau^{*}$ and they are also eigenvalues of $A^{*}$ since $A\left(\tau^{*}\right)$ and $A^{*}$ are a pair of adjoint operators. Let $P$ and $P^{*}$ be the center spaces, that is, the generalized eigenspaces of $A\left(\tau^{*}\right)$ and $A^{*}$ associated with $\Lambda_{0}$, respectively. Then $P^{*}$ is the adjoint space of $P$ and $\operatorname{dim} P=\operatorname{dim} P^{*}=2$. In addition, some easy computations give the following result.

Lemma 7. Let

$$
\begin{aligned}
\sigma & =-\frac{A m \beta^{2}+\left(i \omega_{0}+\mu\right)(m \beta+\alpha+\mu)^{2}}{(m \beta+\mu+\alpha)^{2}\left(\mu+i \omega+\alpha e^{-i \omega_{0} \tau^{*}}\right)}, \\
\sigma^{*} & =\frac{A m \beta^{2}[m \beta+2(\alpha+\mu)]+(\alpha+\mu)(\beta m+\alpha+\mu)^{2}}{(\beta m+\alpha+\mu)\left[A m \beta^{2}+(\alpha+\mu)(m \beta+\mu+\alpha)\left(-\mu-\alpha+i \omega+\alpha e^{-i \omega_{0} \tau^{*}}\right)\right]} .
\end{aligned}
$$


Then

$$
\begin{aligned}
& p_{1}(\theta)=e^{i \omega_{0} \tau^{*} \theta}(1, \sigma)^{T}, \\
& p_{2}(\theta)=\overline{p_{1}(\theta)},
\end{aligned}
$$$$
-1 \leq \theta \leq 0
$$

is a basis of $P$ associated with $\Lambda_{0}$ and

$$
\begin{aligned}
& q_{1}(s)=\left(1, \sigma^{*}\right) e^{-i \omega_{0} \tau^{*} s}, \\
& q_{2}(s)=\overline{q_{1}(s)},
\end{aligned}
$$

is a basis of $Q$ associated with $\Lambda_{0}$.

Let $\Phi=\left(\Phi_{1}, \Phi_{2}\right)$ and $\Psi^{*}=\left(\Psi_{1}^{*}, \Psi_{2}^{*}\right)^{T}$, where

$$
\begin{aligned}
& \Phi_{1}(\theta)=\frac{p_{1}(\theta)+p_{2}(\theta)}{2}=\left(\begin{array}{c}
\operatorname{Re}\left\{e^{i \omega_{0} \tau^{*} \theta}\right\} \\
\operatorname{Re}\left\{\sigma e^{i \omega_{0} \tau^{*} \theta}\right\}
\end{array}\right) \\
& =\left(\begin{array}{c}
\cos \omega_{0} \tau^{*} \theta \\
-\frac{\cos \omega_{0} \tau^{*} \theta\left[\omega_{0}\left(\omega_{0}-\alpha \sin \omega_{0} \tau^{*}\right)+\mu\left(\mu+\alpha \cos \omega_{0} \tau^{*}\right)\right]-\sin \omega_{0} \tau^{*} \theta\left[\omega_{0}\left(\mu+\alpha \cos \omega_{0} \tau^{*}\right)-\mu\left(\omega_{0}-\alpha \sin \omega_{0} \tau^{*}\right)\right]}{\left(\mu+\alpha \cos \omega_{0} \tau^{*}\right)^{2}+\left(\omega_{0}-\alpha \sin \omega_{0} \tau^{*}\right)^{2}}
\end{array}\right), \\
& \Phi_{2}(\theta)=\frac{p_{1}(\theta)-p_{2}(\theta)}{2 i}=\left(\begin{array}{c}
\operatorname{Im}\left\{e^{i \omega_{0} \tau^{*} \theta}\right\} \\
\operatorname{Im}\left\{\sigma e^{i \omega_{0} \tau^{*} \theta}\right\}
\end{array}\right) \\
& =\left(\begin{array}{c}
\sin \omega_{0} \tau^{*} \theta \\
-\frac{\sin \omega_{0} \tau^{*} \theta\left[\omega_{0}\left(\omega_{0}-\alpha \sin \omega_{0} \tau^{*}\right)+\mu\left(\mu+\alpha \cos \omega_{0} \tau^{*}\right)\right]+\cos \omega_{0} \tau^{*} \theta\left[\omega_{0}\left(\mu+\alpha \cos \omega_{0} \tau^{*}\right)-\mu\left(\omega_{0}-\alpha \sin \omega_{0} \tau^{*}\right)\right]}{\left(\mu+\alpha \cos \omega_{0} \tau^{*}\right)^{2}+\left(\omega_{0}-\alpha \sin \omega_{0} \tau^{*}\right)^{2}}
\end{array}\right)
\end{aligned}
$$

\section{for $\theta \in[-1,0]$, and}

$$
\begin{aligned}
& \Psi_{1}^{*}(s)=\frac{q_{1}(s)+q_{2}(s)}{2}=\left(\begin{array}{c}
\operatorname{Re}\left\{e^{-i \omega_{0} \tau^{*} s}\right\} \\
\operatorname{Re}\left\{\sigma^{*} e^{-i \omega_{0} \tau^{*} s}\right\}
\end{array}\right)^{T} \\
& =\left(\begin{array}{c}
\cos \omega_{0} \tau^{*} s \\
-\frac{B_{1} \cos \omega_{0} \tau^{*} s+B_{2} \sin \omega_{0} \tau^{*} s}{(m \beta+\mu+\alpha)\left\{\left[A m \beta^{2}+(\mu+\alpha)(m \beta+\mu+\alpha)\left(-\mu-\alpha+\alpha \cos \omega_{0} \tau^{*}\right)\right]^{2}+(\mu+\alpha)^{2}(m \beta+\alpha+\mu)^{2}\left(\omega_{0}-\alpha \sin \omega_{0} \tau^{*}\right)^{2}\right\}}
\end{array}\right)^{T}, \\
& \Psi_{2}^{*}(s)=\frac{q_{1}(s)-q_{2}(s)}{2 i}=\left(\begin{array}{c}
\operatorname{Im}\left\{e^{-i \omega_{0} \tau^{*} s}\right\} \\
\operatorname{Im}\left\{\sigma^{*} e^{-i \omega_{0} \tau^{*} s}\right\}
\end{array}\right)^{T} \\
& =\left(\begin{array}{c}
-\frac{\sin \omega_{0} \tau^{*} s}{(m \beta+\mu+\alpha)\left\{\left[A m \beta^{2}+(\mu+\alpha)(m \beta+\mu+\alpha)\left(-\mu-\alpha+\alpha \cos \omega_{0} \tau^{*}\right)\right]^{2}+(\mu+\alpha)^{2}(m \beta+\alpha+\mu)^{2}\left(\omega_{0}-\alpha \sin \omega_{0} \tau^{*}\right)^{2}\right\}}
\end{array}\right)^{T},
\end{aligned}
$$

for $s \in[0,1]$, where

$$
\begin{aligned}
B_{1} & =\left\{A m \beta^{2}[m \beta+2(\mu+\alpha)]\right. \\
& \left.+(\mu+\alpha)^{2}(m \beta+\alpha+\mu)^{2}\right\}\left[A m \beta^{2}\right. \\
& \left.+(\alpha+\mu)(\beta m+\mu+\alpha)\left(-\mu-\alpha+\alpha \cos \omega_{0} \tau^{*}\right)\right] \\
& +\omega_{0}(\mu+\alpha)^{*}(\beta m+\mu+\alpha)^{3}\left(\omega_{0}-\alpha \sin \omega_{0} \tau^{*}\right),
\end{aligned}
$$

$$
\begin{aligned}
B_{2} & =-(\mu+\alpha)(\beta m+\alpha+\mu)\left(\omega_{0}-\alpha \sin \omega_{0} \tau^{*}\right) \\
& \cdot\left[A m \beta^{2}(\beta m+2 \mu+2 \alpha)\right. \\
& \left.+(\mu+\alpha)^{2}(\beta m+\alpha+\mu)^{2}\right]+\omega_{0}(\mu+\alpha)(m \beta+\alpha \\
& +\mu)^{2}\left[A m \beta^{2}\right. \\
& \left.+(\mu+\alpha)(\beta m+\mu+\alpha)\left(-\mu-\alpha+\alpha \cos \omega_{0} \tau^{*}\right)\right] .
\end{aligned}
$$



that

From (33), we can obtain $\left(\Psi_{1}^{*}, \Phi_{1}\right)$ and $\left(\Psi_{1}^{*}, \Phi_{2}\right)$. Noting

$$
\begin{aligned}
\left(q_{1}, p_{1}\right)= & \left(\Psi_{1}^{*}, \Phi_{1}\right)-\left(\Psi_{2}^{*}, \Phi_{2}\right) \\
& +i\left[\left(\Psi_{1}^{*}, \Phi_{2}\right)+\left(\Psi_{2}^{*}, \Phi_{1}\right)\right], \\
\left(q_{1}, p_{1}\right)= & 1+\sigma \sigma^{*}-\tau^{*} e^{-i \omega_{0} \tau^{*}} \alpha \sigma \sigma^{*}:=D^{*} .
\end{aligned}
$$

Therefore, we can obtain

$$
\begin{aligned}
& \left(\Psi_{1}^{*}, \Phi_{1}\right)-\left(\Psi_{2}^{*}, \Phi_{2}\right)=\operatorname{Re}\left\{D^{*}\right\}, \\
& \left(\Psi_{1}^{*}, \Phi_{2}\right)+\left(\Psi_{2}^{*}, \Phi_{1}\right)=\operatorname{Im}\left\{D^{*}\right\} .
\end{aligned}
$$

Now, we define $\left(\Psi^{*}, \Phi\right)=\left(\Psi_{j}^{*}, \Phi_{k}\right)(j, k=1,2)$ and construct a new basis $\psi$ for $Q$ by $\Psi=\left(\Psi_{1}, \Psi_{2}\right)^{T}=$ $\left(\Psi^{*}, \Phi\right)^{-1} \Psi^{*}$. Obviously, $(\Psi, \Phi)=I_{2}$, the second order identity matrix. In addition, define $f=\left(\xi^{1}, \xi^{2}\right)$ and $c \cdot f=$ $c_{1} \xi^{1}+c_{2} \xi^{2}$ for $c=\left(c_{1}, c_{2}\right)^{T}, c_{j} \in R(j=1,2)$. Then the center space of linear equation (30) is given by $P_{C N} \mathscr{C}$, where

$$
P_{\mathrm{CN}} \varphi=\Phi(\Psi,\langle\varphi, f\rangle) \cdot f, \quad \varphi \in \mathscr{C},
$$

and $\mathscr{C}=P_{\mathrm{CN}} \mathscr{C} \oplus P_{S} \mathscr{C}$; here $P_{S} \mathscr{C}$ denote the complementary subspace of $P_{C N} \mathscr{C}$.

Let $A_{\tau^{*}}$ be defined by

$$
\begin{array}{r}
A_{\tau^{*}} \varphi(\theta)=\dot{\varphi}(\theta)+X_{0}(\theta)\left[L\left(\tau^{*}\right)(\varphi(\theta))-\dot{\varphi}(0)\right], \\
\varphi \in B \mathscr{C},
\end{array}
$$

where

$$
X_{0}(\theta)= \begin{cases}0, & -1 \leq \theta<0 \\ I, & \theta=0 .\end{cases}
$$

Then $A_{\tau^{*}}$ is the infinitesimal generator induced by the solution of (30) and (28) can be rewritten as the following operator differential equation:

$$
\dot{U}_{t}=A_{\tau^{*}} U_{t}+X_{0} F\left(U_{t}, \mu\right) .
$$

Using the decomposition $\mathscr{C}=P_{\mathrm{CN}} \mathscr{C} \oplus P_{S} \mathscr{C}$ and (43), the solution of (46) can be rewritten as

$$
U_{t}=\Phi\left(\begin{array}{l}
x_{1}(t) \\
x_{2}(t)
\end{array}\right) \cdot f+h\left(x_{1}, x_{2}, \gamma\right)
$$

where

$$
\left(\begin{array}{l}
x_{1}(t) \\
x_{2}(t)
\end{array}\right)=\left(\Psi,\left\langle U_{t}, f\right\rangle\right)
$$

and $h\left(x_{1}, x_{2}, \gamma\right) \in P_{s} c$ with $h(0,0,0)=D h(0,0,0)=0$. In particular, the solution of (28) on the center manifold is given by

$$
U_{t}^{*}=\Phi\left(\begin{array}{l}
x_{1}(t) \\
x_{2}(t)
\end{array}\right) \cdot f+h\left(x_{1}, x_{2}, 0\right) .
$$

Setting $z=x_{1}-i x_{2}$ and noticing that $p_{1}=\Phi_{1}+i \Phi_{2}$, then (49) can be rewritten as

$$
\begin{aligned}
U_{t}^{*} & =\frac{1}{2} \Phi\left(\begin{array}{c}
z+\bar{z} \\
i(z-\bar{z})
\end{array}\right) \cdot f+w(z, \bar{z}) \\
& =\frac{1}{2}\left(p_{1} z+\overline{p_{1} z}\right) \cdot f+W(z, \bar{z}),
\end{aligned}
$$

where $W(z, \bar{z})=h((z+\bar{z}) / 2,-(z-\bar{z}) / 2 i, 0)$. Moreover, by [17], $z$ satisfies

$$
\dot{z}=i \omega_{0} \tau^{*} z+g(z, \bar{z})
$$

where

$$
g(z, \bar{z})=\left(\Psi_{1}(0)-i \Psi_{2}(0)\right)\left\langle F\left(U_{t}^{*}, 0\right), f\right\rangle .
$$

Let

$$
\begin{gathered}
W(z, \bar{z})=W_{20} \frac{z^{2}}{2}+W_{11} z \bar{z}+W_{02} \frac{\bar{z}^{2}}{2}+\cdots, \\
g(z, \bar{z})=g_{20} \frac{z^{2}}{2}+g_{11} z \bar{z}+g_{02} \frac{\bar{z}^{2}}{2}+\cdots
\end{gathered}
$$

According to (50) we have

$$
\begin{gathered}
\left\langle F\left(U_{t}^{*}, 0\right), f\right\rangle=\frac{\tau^{*}}{4}\left(\left[-\beta \sigma+\frac{m A \beta^{3}}{(m \beta+\mu+\alpha)^{3}}\right] z^{2}+\left[\begin{array}{c}
\left.-\beta \sigma+\frac{2 m A \beta^{3}}{(m \beta+\mu+\alpha)^{3}}-\beta \bar{\sigma}\right] z \bar{z}+\left[\frac{m A \beta^{3}}{(m \beta+\mu+\alpha)^{3}}-\beta \bar{\sigma}\right] \bar{z}^{2} \\
\beta \sigma z^{2}+\beta(\sigma+\bar{\sigma}) z \bar{z}+\beta \bar{\sigma} \bar{z}^{2}
\end{array}\right)+\frac{\tau^{*}}{4}\right. \\
\times\left(\begin{array}{c}
\left\langle-\beta\left(2 W_{11}^{(2)}(0)+W_{20}^{(2)}(0)+\bar{\sigma} W_{20}^{(1)}(0)+2 \sigma W_{11}^{(1)}(0)\right)+\frac{2 m A \beta^{3}}{(m \beta+\mu+\alpha)^{3}}\left(2 W_{11}^{(1)}(0)+W_{20}^{(1)}(0)\right)-\frac{3 m A \beta^{4}}{2(m \beta+\alpha+\mu)^{4}}, 1\right\rangle \\
\left\langle\beta\left(2 W_{11}^{(2)}(0)+W_{20}^{(2)}(0)+\bar{\sigma} W_{20}^{(1)}(0)+2 \sigma W_{11}^{(1)}(0)\right), 1\right\rangle
\end{array}\right) z^{2} \bar{z}
\end{gathered}
$$


where

$$
\begin{aligned}
&\left\langle W_{i j}^{(n)}(\theta), 1\right\rangle=\frac{1}{\pi} \int_{0}^{\pi} W_{i j}^{(n)}(\theta)(x) d x \\
& i+j=2, n=1,2,3 .
\end{aligned}
$$

Let $\left(\psi_{1}, \psi_{2}\right)=\Psi_{1}(0)-i \Psi_{2}(0)$. Therefore, we obtain

$$
\begin{aligned}
& g_{20}=\frac{\tau^{*}}{2}\left\{\left[-\beta \sigma+\frac{m A \beta^{3}}{(m \beta+\mu+\alpha)^{3}}\right] \psi_{1}+\beta \sigma \psi_{2}\right\} \\
& g_{11}=\frac{\tau^{*}}{4}\left\{\left[-\beta \sigma-\beta \bar{\sigma}+\frac{2 m A \beta^{3}}{(m \beta+\mu+\alpha)^{3}}\right] \psi_{1}+\beta(\sigma\right. \\
& \left.+\bar{\sigma}) \psi_{2}\right\}, \\
& g_{02}=\frac{\tau^{*}}{2}\left\{\left[-\beta \bar{\sigma}+\frac{m A \beta^{3}}{(m \beta+\mu+\alpha)^{3}}\right] \psi_{1}+\beta \bar{\sigma} \psi_{2}\right\} \\
& g_{21}=\frac{\tau^{*}}{2}\left[\left\langle-\beta\left(2 W_{11}^{(2)}(0)+W_{20}^{(2)}(0)+\bar{\sigma} W_{20}^{(1)}(0)\right.\right.\right. \\
& \left.+2 \sigma W_{11}^{(1)}(0)\right)+\frac{2 m A \beta^{3}}{(m \beta+\mu+\alpha)^{3}}\left(2 W_{11}^{(1)}(0)\right. \\
& \left.\left.\left.+2 \sigma W_{11}^{(1)}(0)\right), 1\right\rangle \psi_{2}\right] . \\
& \left.\left.+W_{20}^{(1)}(0)\right)-\frac{3 m A \beta^{4}}{2(m \beta+\alpha+\mu)^{4}}, 1\right\rangle \psi_{1} \\
& +\left\langle\beta \left( 2 W_{11}^{(2)}(0)+W_{20}^{(2)}(0)+\bar{\sigma} W_{20}^{(1)}(0)\right.\right.
\end{aligned}
$$

Since $W_{20}(\theta), W_{11}(\theta)$ for $\theta \in[-1,0]$ appear in $g_{21}$, we still need to compute them. It follows easily from (53) that

$$
\begin{gathered}
\dot{W}(z, \bar{z})=W_{20} z \dot{z}+W_{11}(\dot{z} \bar{z}+z \overline{\dot{z}})+W_{02} \bar{z} \overline{\dot{z}}+\cdots \\
A_{\tau^{*}} W=A_{\tau^{*}} W_{20} \frac{z^{2}}{2}+A_{\tau^{*}} W_{11} z \bar{z}+A_{\tau^{*}} W_{02} \frac{\bar{z}^{2}}{2}
\end{gathered}
$$

In addition, by [17], $W(z(t), \bar{z}(t))$ satisfies

$$
\dot{W}=A_{\tau^{*}} W+H(z, \bar{z}),
$$

where

$$
\begin{aligned}
H(z, \bar{z})= & H_{20} \frac{z^{2}}{2}+H_{11} z \bar{z}+H_{02} \frac{\bar{z}^{2}}{2}+\cdots \\
= & X_{0} F\left(U_{t}^{*}, 0\right)-\Phi\left(\Psi,\left\langle X_{0} F\left(U_{t}^{*}, 0\right), f\right\rangle\right) \\
& \cdot f
\end{aligned}
$$

with $H_{i j} \in P_{S} \mathscr{C}, i+j=2$.
Thus, from (51) and (58)-(60), we can obtain

$$
\begin{aligned}
\left(2 i \omega_{0} \tau^{*}-A_{\tau^{*}}\right) W_{20} & =H_{20}, \\
-A_{\tau^{*}} W_{11} & =H_{11} .
\end{aligned}
$$

Noticing that $A_{\tau^{*}}$ has only two eigenvalues $\pm i \omega_{0} \tau^{*}$ with zero real parts, therefore, (59) has unique solution $W_{i j}(i+j=$ 2) in $P_{S} \mathscr{C}$ given by

$$
\begin{aligned}
& W_{20}=\left(2 i \omega_{0} \tau^{*}-A_{\tau^{*}}\right)^{-1} H_{20}, \\
& W_{11}=-A_{\tau^{*}}^{-1} H_{11} .
\end{aligned}
$$

From (60), we know that, for $-1 \leq \theta<0$,

$H(z, \bar{z})=-\Phi(\theta) \Psi(0)\left\langle F\left(U_{t}^{*}, 0\right), f_{0}\right\rangle \cdot f_{0}$,

$$
\begin{aligned}
= & -\left(\frac{p_{1}(\theta)+p_{2}(\theta)}{2}, \frac{p_{1}(\theta)-p_{2}(\theta)}{2 i}\right) \\
& \cdot\left(\Psi_{1}(0) \Psi_{2}(0)\right) \times\left\langle F\left(U_{t}^{*}, 0\right), f_{0}\right\rangle \cdot f_{0}, \\
= & -\frac{1}{2}\left[p_{1}(\theta)\left(\Psi_{1}(0)-i \Psi_{2}(0)\right)\right. \\
& \left.+p_{2}(\theta)\left(\Psi_{1}(0)+i \Psi_{2}(0)\right)\right] \times\left\langle F\left(U_{t}^{*}, 0\right), f_{0}\right\rangle \cdot f_{0}, \\
= & -\frac{1}{4}\left[g_{20} p_{1}(\theta)+\bar{g}_{02} p_{2}(\theta)\right] z^{2} \cdot f_{0}-\frac{1}{2}\left[g_{11} p_{1}(\theta)\right. \\
& \left.+\bar{g}_{11} p_{2}(\theta)\right] z \bar{z} \cdot f_{0}+\cdots .
\end{aligned}
$$

Therefore, for $-1 \leq \theta<0$,

$$
\begin{aligned}
H_{20}(\theta)= & -\frac{1}{2}\left[g_{20} p_{1}(\theta)+\bar{g}_{02} p_{2}(\theta)\right] \cdot f, \\
H_{11}(\theta)= & -\frac{1}{2}\left[g_{11} p_{1}(\theta)+\bar{g}_{11} p_{2}(\theta)\right] \cdot f, \\
H(z, \bar{z})(0)= & F\left(U_{t}^{*}, 0\right)-\Phi\left(\Psi,\left\langle F\left(U_{t}^{*}, 0\right), f\right\rangle\right) \cdot f, \\
H_{20}(0)= & \frac{\tau^{*}}{2}\left(\begin{array}{c}
\left.-\beta \sigma+\frac{m A \beta^{3}}{(m \beta+\mu+\alpha)^{3}}\right) \\
\beta \sigma
\end{array}\right) \\
& -\frac{1}{2}\left[g_{20} p_{1}(0)+\bar{g}_{02} p_{2}(0)\right] \cdot f, \\
H_{11}(0)= & \frac{\tau^{*}}{4}\left(\begin{array}{c}
-\beta \sigma-\beta \bar{\sigma}+\frac{2 m A \beta^{3}}{(m \beta+\mu+\alpha)^{3}} \\
\beta(\sigma+\bar{\sigma})
\end{array}\right) \\
& -\frac{1}{2}\left[g_{11} p_{1}(0)+\bar{g}_{11} p_{2}(0)\right] \cdot f .
\end{aligned}
$$

By the definition of $A_{\tau^{*}}$, we get from (62) that

$\dot{W}_{20}(\theta)=2 i \omega_{0} \tau^{*} \mathrm{~W}_{20}(\theta)+\frac{1}{2}\left[g_{20} p_{1}(\theta)+\bar{g}_{02} p_{2}(\theta)\right]$ 
Noting that $p_{1}(\theta)=p_{1}(0) e^{i \omega_{0} \tau^{*}},-1 \leq \theta \leq 0$,

$$
\begin{aligned}
W_{20}(\theta)= & \frac{i}{2}\left[\frac{g_{20}}{\omega_{0} \tau^{*}} p_{1}(\theta)+\frac{\bar{g}_{02}}{3 \omega_{0} \tau^{*}} p_{2}(\theta)\right] \cdot f_{0} \\
& +E e^{2 i \omega_{0} \tau^{*} \theta} \\
E= & W_{20}(0)-\frac{i}{2}\left[\frac{g_{20}}{\omega_{0} \tau^{*}} p_{1}(0)+\frac{\bar{g}_{02}}{3 \omega_{0} \tau^{*}} p_{2}(0)\right] \\
& \cdot f .
\end{aligned}
$$

Using the definition of $A_{\tau^{*}}$ and combining (62) and (67), we get

$$
\begin{array}{r}
2 i \omega_{0} \tau^{*}\left[\frac{i g_{20}}{2 \omega_{0} \tau^{*}} p_{1}(0) \cdot f+\frac{i \bar{g}_{02}}{6 \omega_{0} \tau^{*}} p_{2}(0) \cdot f+E\right] \\
-L\left(\tau^{*}\right)\left[\frac{i g_{20}}{2 \omega_{0} \tau^{*}} p_{1}(\theta) \cdot f+\frac{i \bar{g}_{02}}{6 \omega_{0} \tau^{*}} p_{2}(\theta) \cdot f\right.
\end{array}
$$

$$
E=\frac{1}{2}\left(\begin{array}{c}
2 i \omega_{0}+\frac{A m \beta^{2}}{\beta m+\mu+\alpha}\left(\frac{1}{\beta m+\mu+\alpha}+\frac{1}{\mu+\alpha}\right) \\
-\frac{A m \beta^{2}}{(\beta m+\mu+\alpha)(\mu+\alpha)}+\mu
\end{array}\right.
$$

By a similar way, we have

$$
\begin{aligned}
& \dot{W}_{11}(\theta)=\frac{1}{2}\left[g_{11} p_{1}(\theta)+\bar{g}_{11} p_{2}(\theta)\right] \cdot f, \quad-1 \leq \theta<0, \\
& W_{11}(\theta)=\frac{i}{2 \omega_{0} \tau^{*}}\left[g_{11} p_{1}(\theta)-\bar{g}_{11} p_{2}(\theta)\right] \cdot f+F .
\end{aligned}
$$

Similar to the above, we can obtain

F

$$
\begin{aligned}
& =\frac{1}{4}\left(\begin{array}{cc}
\frac{A m \beta^{2}}{\beta m+\mu+\alpha}\left(\frac{1}{\beta m+\mu+\alpha}+\frac{1}{\mu+\alpha}\right) & \alpha+\mu \\
-\frac{A m \beta^{2}}{(\beta m+\mu+\alpha)(\mu+\alpha)}+\mu & 0
\end{array}\right)^{-1} \\
& \quad \times\left(\begin{array}{c}
-\beta \sigma-\beta \bar{\sigma}+\frac{2 m A \beta^{3}}{(m \beta+\mu+\alpha)^{3}} \\
\beta \sigma+\beta \bar{\sigma}
\end{array}\right) .
\end{aligned}
$$

Theorem 8. System (3) has the following Poincaré normal form:

$$
\dot{\xi}=i \omega_{0} \tau^{*} \xi+c_{1}(0) \xi|\xi|^{2}+o\left(|\xi|^{5}\right)
$$

where

$$
c_{1}(0)=\frac{i}{2 \omega_{0} \tau^{*}}\left[g_{20} g_{11}-2\left|g_{11}\right|^{2}-\frac{\left|g_{02}\right|^{2}}{3}\right]+\frac{g_{21}}{2},
$$

$$
\begin{aligned}
& \left.+E e^{2 i \omega_{0} \tau^{*} \theta}\right]=\frac{\tau^{*}}{2}\left(\begin{array}{c}
-\beta \sigma+\frac{m A \beta^{3}}{(m \beta+\alpha+\mu)^{3}} \\
\beta \sigma
\end{array}\right) \\
& -\frac{1}{2}\left[g_{20} p_{1}(0)+\bar{g}_{02} p_{2}(0)\right] \cdot f .
\end{aligned}
$$

From

$$
\begin{aligned}
& L\left(\tau^{*}\right)\left[p_{1}(\theta) \cdot f\right]=i \omega_{0} \tau^{*} p_{1}(0) \cdot f, \\
& L\left(\tau^{*}\right)\left[p_{2}(\theta) \cdot f\right]=-i \omega_{0} \tau^{*} p_{2}(0) \cdot f,
\end{aligned}
$$

we get

$$
\begin{aligned}
& 2 i \omega_{0} \tau^{*} E-L\left(\tau^{*}\right)\left(E e^{2 i \omega_{0} \tau^{*} \theta}\right) \\
& =\frac{\tau^{*}}{2}\left(\begin{array}{c}
-\beta \sigma+\frac{m A \beta^{3}}{(m \beta+\mu+\alpha)^{3}} \\
\beta \sigma
\end{array}\right) .
\end{aligned}
$$

From the above expression, we can see easily that

$\left.2 i \omega_{0}-\alpha+\alpha e^{-2 i \omega_{0} \tau^{*}}\right)^{-1} \times\left(\begin{array}{c}-\beta \sigma+\frac{m A \beta^{3}}{(m \beta+\mu+\alpha)^{3}} \\ \beta \sigma\end{array}\right)$.

so we can compute the following result:

$$
\begin{aligned}
& \sigma_{2}=-\frac{\operatorname{Re}\left(c_{1}(0)\right)}{\operatorname{Re}\left(\lambda^{\prime}\left(\tau^{*}\right)\right)}, \\
& \beta_{2}=2 \operatorname{Re}\left(c_{1}(0)\right), \\
& T_{2}=-\frac{\operatorname{Im}\left(c_{1}(0)\right)+\sigma_{2} \operatorname{Im}\left(\lambda^{\prime}\left(\tau^{*}\right)\right)}{\omega_{0} \tau^{*}},
\end{aligned}
$$

which determine the properties of bifurcating periodic solutions at the critical values $\tau^{*}$; that is, $\sigma_{2}$ determines the directions of the Hopf bifurcation: if $\sigma_{2}>0\left(\sigma_{2}<0\right)$, then the Hopf bifurcation is supercritical (subcritical) and the bifurcating periodic solutions exist for $\tau>\tau^{*} ; \beta_{2}$ determines the stability of the bifurcating periodic solutions: the bifurcating periodic solutions on the center manifold are stable (unstable), if $\beta_{2}<$ $0\left(\beta_{2}>0\right)$; and $T_{2}$ determines the period of the bifurcating periodic solutions: the periodic increase (decrease), if $T_{2}>$ $0\left(T_{2}<0\right)$.

\section{Numerical Solutions}

Using MATLAB, numerical simulations are provided to substantiate the theoretical results established in the previous sections of this paper. 


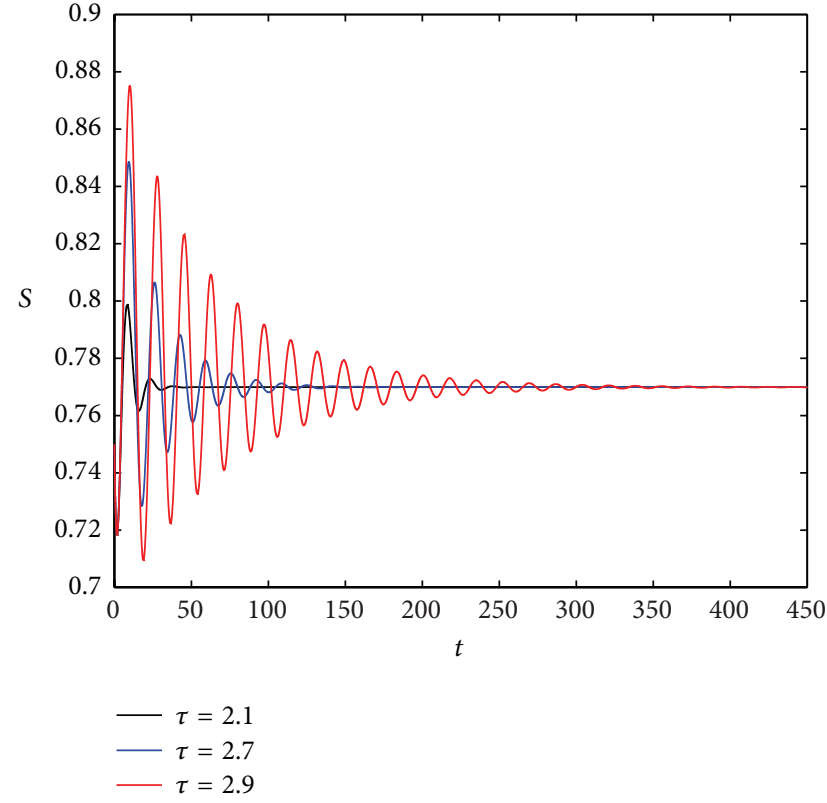

(a)

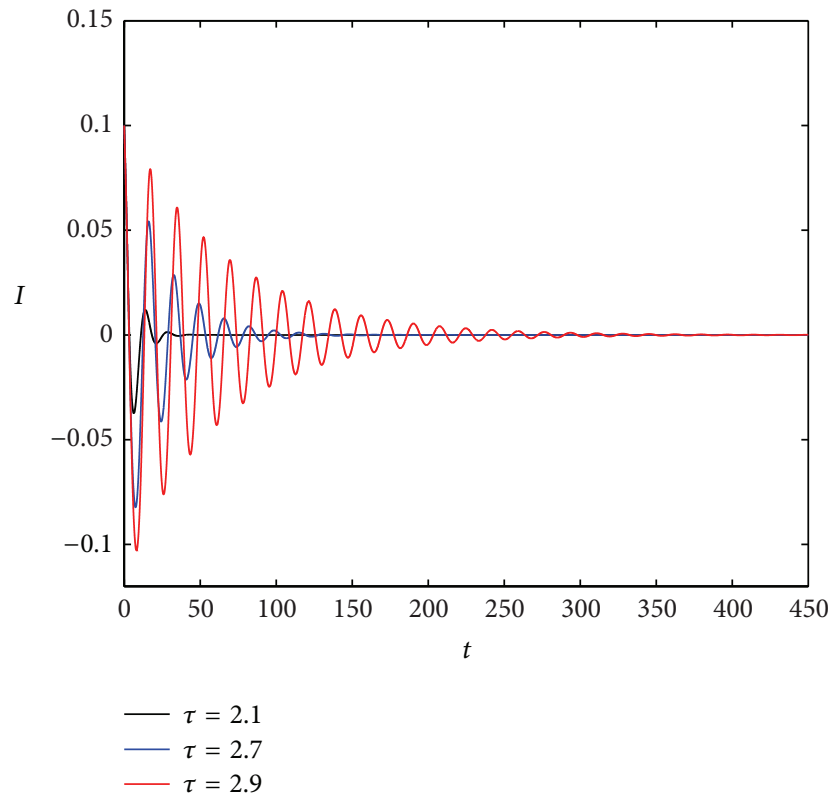

(b)

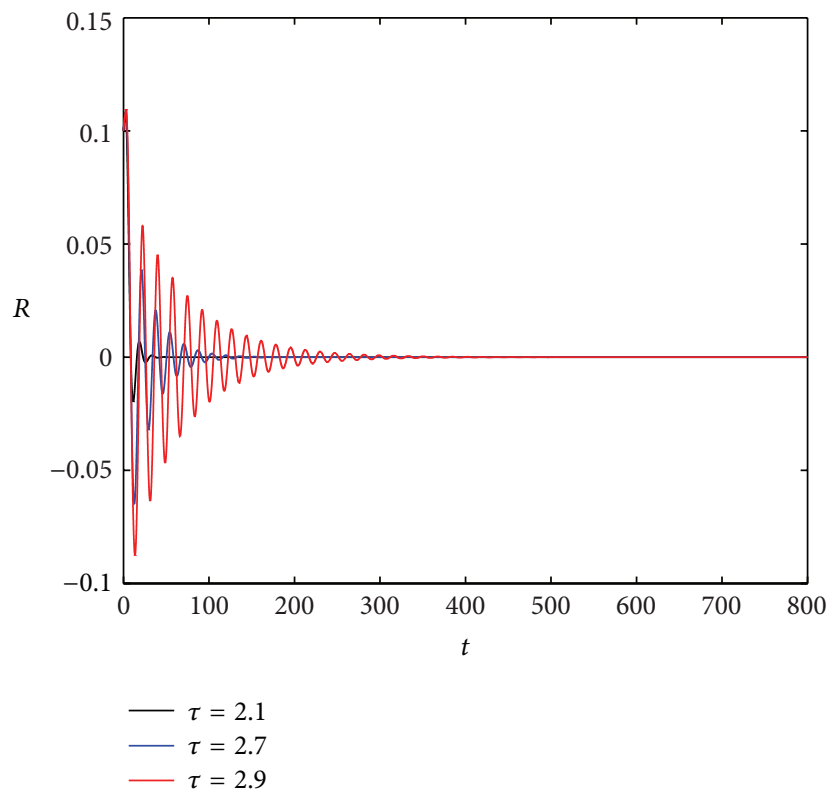

(c)

Figure 1: The boundary equilibrium point $E^{1}$ is locally asymptotically stable as $\tau=2.1,2.7,2.9<\tau_{0}$. (a) The susceptible users; (b) the infected users; (c) the rational users.

Example 1. Choose $A=0.5, m=0.9, \beta=0.7, \alpha=0.4, \mu=$ $0.35, S_{0}=0.75, I_{0}=0.1$, and $R_{0}=0.1$ in system (1). A simple calculation shows that the boundary equilibrium point $E^{1}=$ $(0.7600,0,0)^{T}$. Obviously, the conditions of Theorem 2 satisfy. It is easy to obtain that $\tau_{0}=3.06$. According to Theorem 2 , the boundary equilibrium point $E^{1}$ is locally asymptotically stable for $\tau=2.1,2.7,2.9<\tau_{0}$, as shown in Figure 1. That is to say, at last there are not any rumor infected people and rumor recovered people except rumor susceptible people, and the rumor propagation is controlled effectively. Furthermore, from Figure 1 we can find that as $\tau$ increasing, system (1) takes more time to converge to the boundary equilibrium point $E^{1}$. When $\tau=3.08>\tau_{0}$, a spatially homogeneous periodic solution emerges from the boundary equilibrium point $E^{1}$ (see Figure 2). That is, the boundary equilibrium point $E^{1}$ is unstable, which seriously disrupts the normal social order.

Example 2. Consider system (1) with $A=0.2, m=0.8, \beta=$ $0.5, \alpha=0.2, \mu=0.1, S_{0}=0.61, I_{0}=0.18$, and $R_{0}=0.36$. It is easy to obtain that the positive equilibrium point of system (1) 


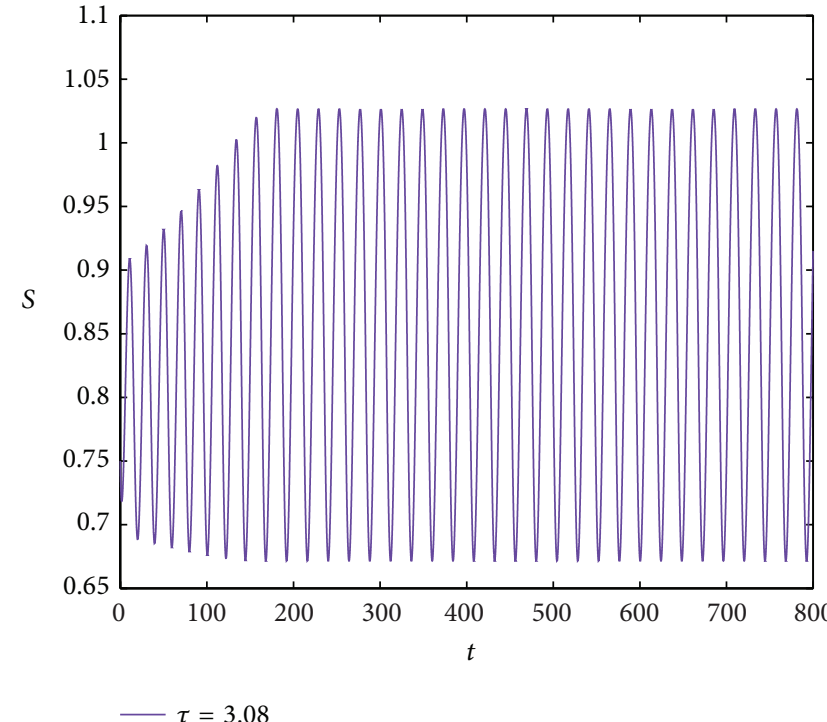

(a)

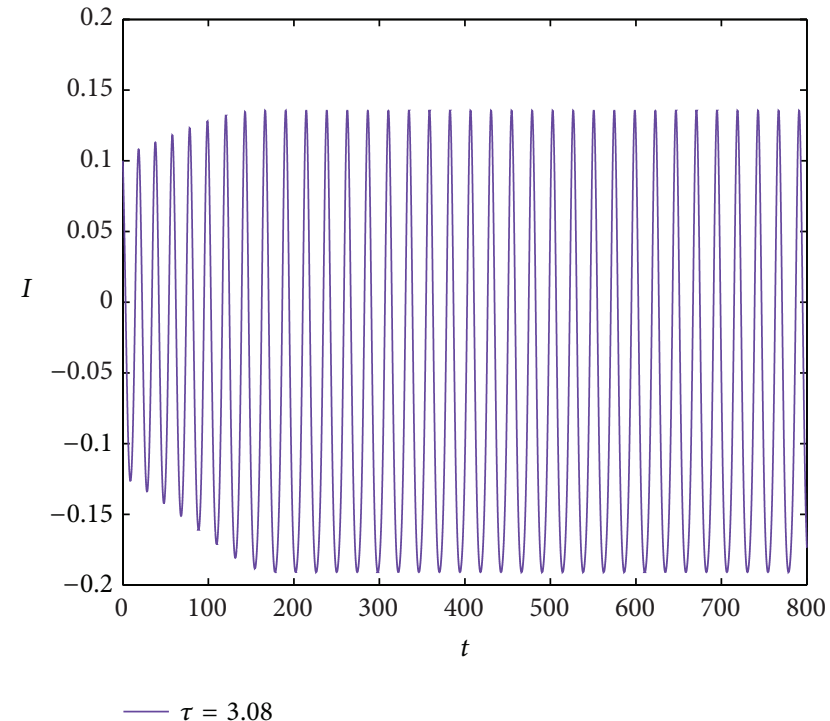

(b)

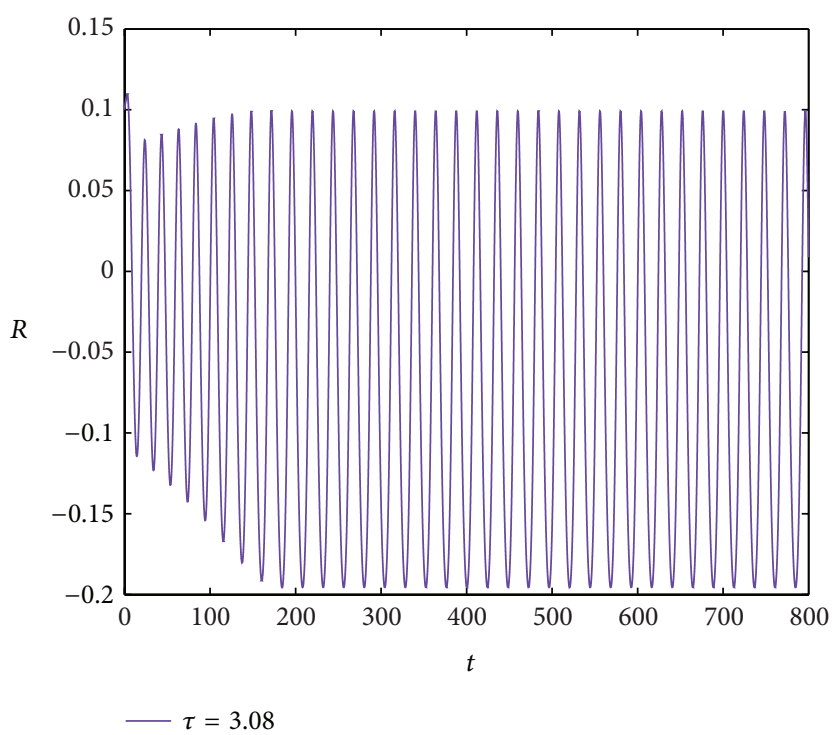

(c)

Figure 2: The periodic oscillation derived from Hopf bifurcation around the boundary equilibrium point $E^{1}$ occurs as $\tau=3.08>\tau_{0}$. (a) The susceptible users; (b) the infected users; (c) the rational users.

is $E^{*}=(0.6000,0.1810,0.3619)^{T}$. Obviously, the conditions of Theorem 6 satisfy. By a simple calculation, we can obtain that $\tau_{0}^{*}=4.2707$. According to Theorem 6, the positive equilibrium point $E^{*}$ is locally asymptotically stable for $\tau=$ $3<\tau_{0}^{*}$, as shown in Figure 3. That is to say, the densities of the rumor susceptible people, the rumor infected people, and the rumor recovered people are controlled in fixed values, which is beneficial in controlling the rumor propagation. When $\tau=4.28>\tau_{0}^{*}$, a spatially homogeneous periodic solution emerges from the positive equilibrium point $E^{*}$ (see Figure 4), which can lead to social instability and disrupt social harmony.

\section{Conclusion}

In this paper, we introduce a delay into rumor propagation in an emergency-affected environment and establish a delayed SIR rumor propagation model. The theoretical analysis and numerical simulation reveal that the discrete delay is responsible for the stability switch of the rumor propagation model, and a Hopf bifurcation occurs as the delay increases through a certain threshold. Furthermore, when the system is stable, as $\tau$ increasing, the system will take more time to converge to the equilibrium state. In future, we will further discuss rumor spreads in space and establish a temporal-spatial rumor propagation model based on the reaction diffusion equation. 


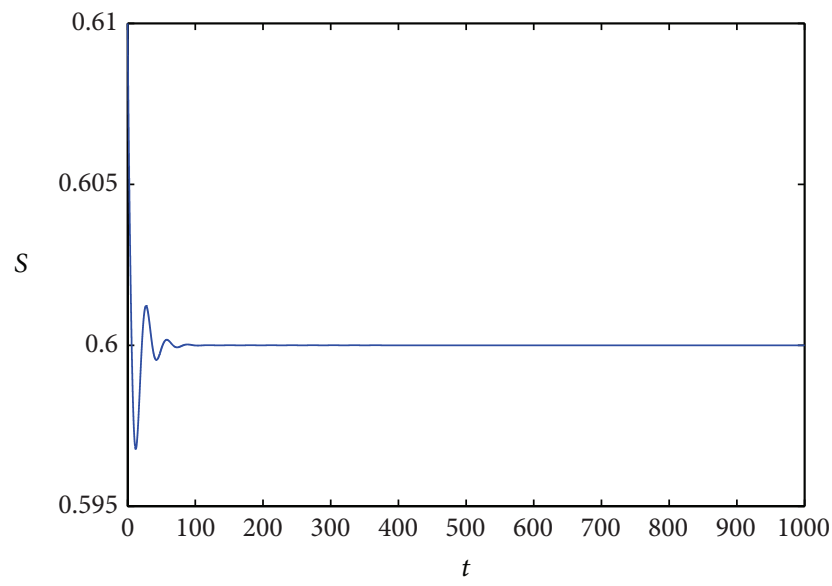

(a)

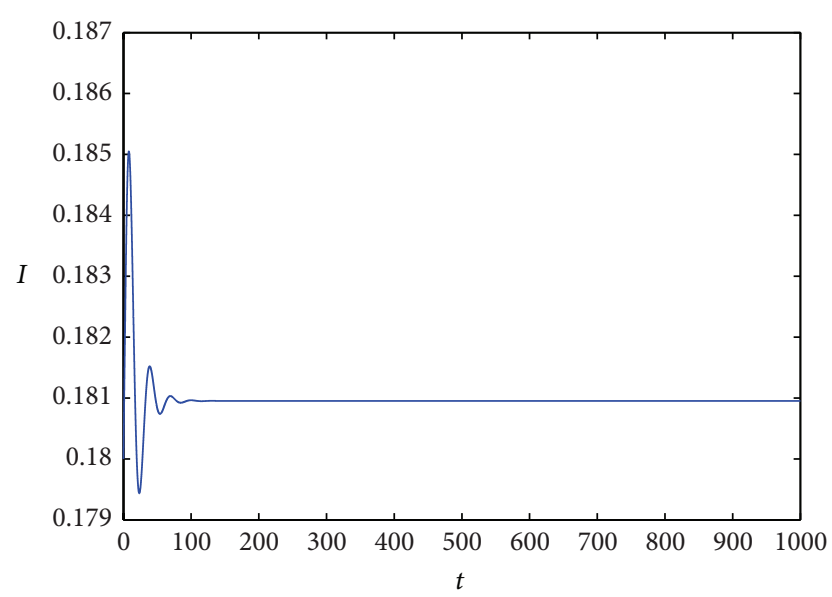

(b)

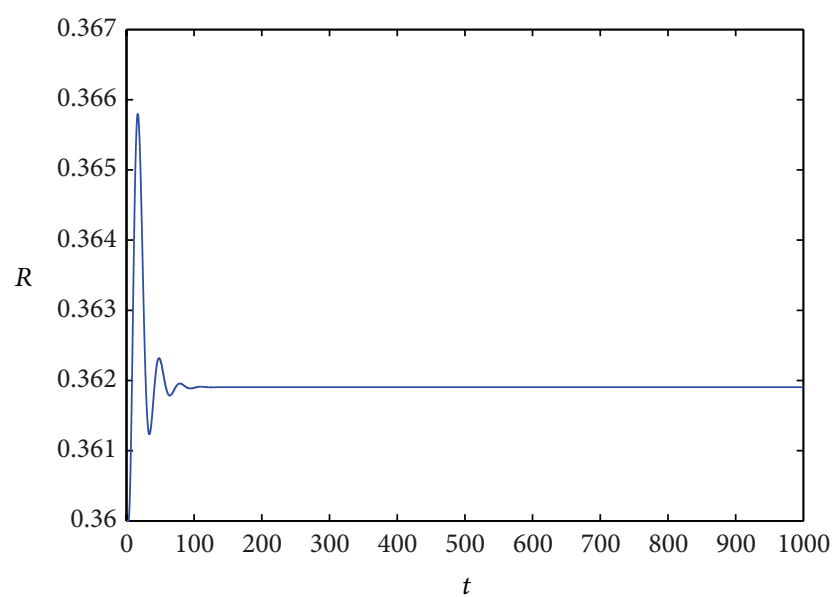

(c)

FIGURE 3: The positive equilibrium point $E^{*}$ is locally asymptotically stable as $\tau=3<\tau_{0}^{*}$. (a) The susceptible users; (b) the infected users; (c) the rational users.

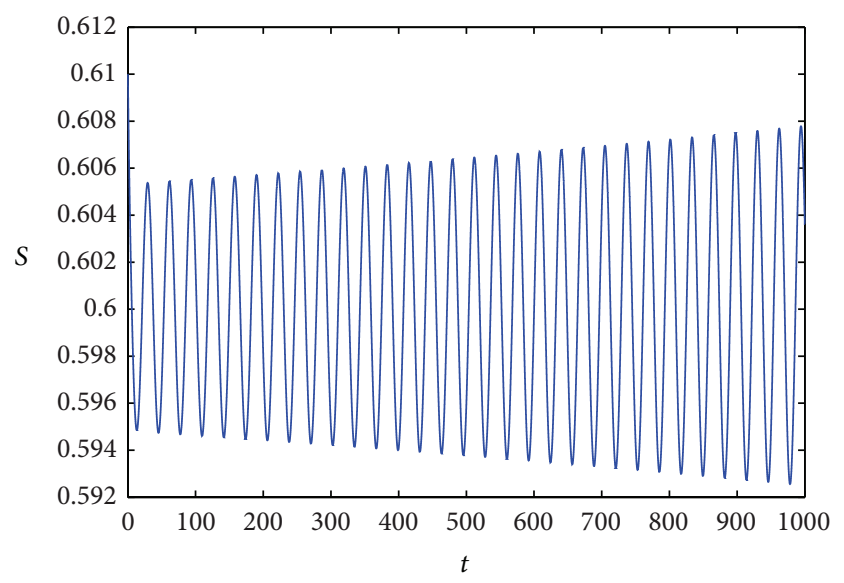

(a)

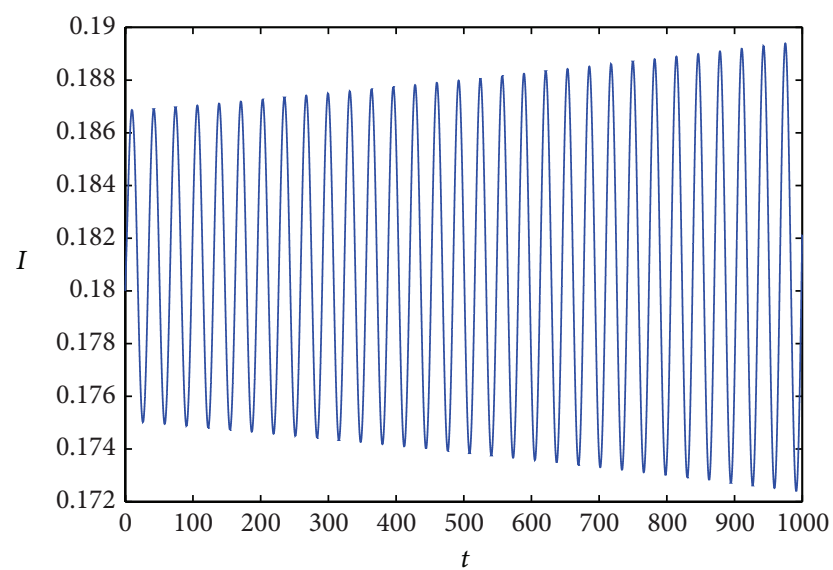

(b)

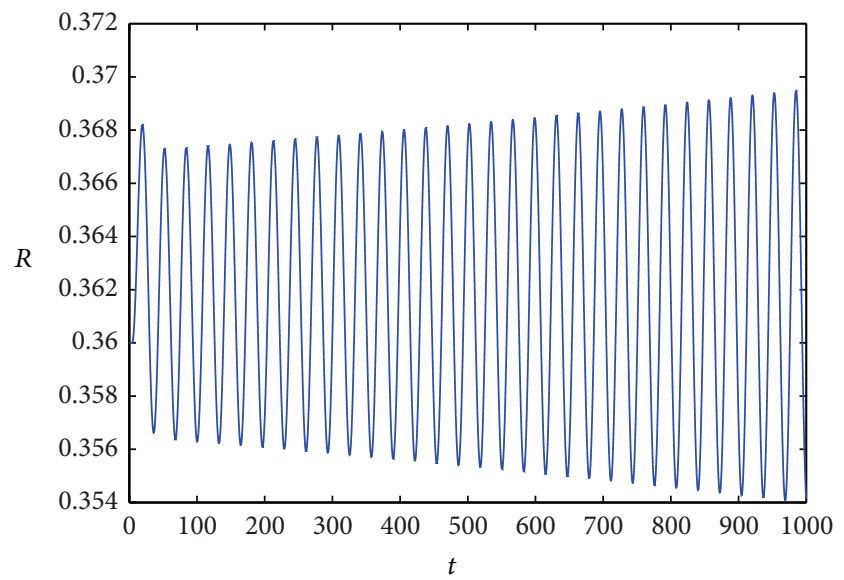

(c)

FIgURE 4: The periodic oscillation derived from Hopf bifurcation around the positive equilibrium point $E^{*}$ occurs as $\tau=4.28>\tau_{0}^{*}$. (a) The susceptible users; (b) the infected users; (c) the rational users. 


\section{Conflict of Interests}

The authors declare that there is no conflict of interests regarding the publication of this paper.

\section{Acknowledgment}

This work is supported by the National Natural Science Foundation of China under Grant no. 90924012.

\section{References}

[1] S. Galam, "Modelling rumors: the no plane Pentagon French hoax case," Physica A: Statistical Mechanics and its Applications, vol. 320, pp. 571-580, 2003.

[2] M. Kosfeld, "Rumours and markets," Journal of Mathematical Economics, vol. 41, no. 6, pp. 646-664, 2005.

[3] H. Hayakawa, Sociology of Rumor-Approach from Formal Sociology, Seikyusya, Tokyo, Japan, 2002.

[4] L. Zhao, H. Cui, X. Qiu, X. Wang, and J. Wang, "Sir rumor spreading model in the new media age," Physica A. Statistical Mechanics and Its Applications, vol. 392, no. 4, pp. 995-1003, 2013.

[5] K. Afassinou, "Analysis of the impact of education rate on the rumor spreading mechanism," Physica A: Statistical Mechanics and Its Applications, vol. 414, pp. 43-52, 2014.

[6] J. Zhou, Z. Liu, and B. Li, "Influence of network structure on rumor propagation," Physics Letters A, vol. 368, no. 6, pp. 458463, 2007.

[7] L. Zhao, X. Qiu, X. Wang, and J. Wang, "Rumor spreading model considering forgetting and remembering mechanisms in inhomogeneous networks," Physica A: Statistical Mechanics and Its Applications, vol. 392, no. 4, pp. 987-994, 2013.

[8] F. Roshani and Y. Naimi, "Effects of degree-biased transmission rate and nonlinear infectivity on rumor spreading in complex social networks," Physical Review E, vol. 85, no. 3, Article ID 036109, 2012.

[9] Y. Zhang, S. Zhou, Z. Zhang, J. Guan, and S. Zhou, "Rumor evolution in social networks," Physical Review E, vol. 87, no. 3, Article ID 032133, 2013.

[10] D. J. Daley and D. G. Kendall, "Stochastic rumours," IMA Journal of Applied Mathematics, vol. 1, no. 1, pp. 42-55, 1965.

[11] D. Maki and M. Thompson, Mathematical Models and Applications: With Emphasis on the Social, Life, and Management Sciences, AMC, 1973.

[12] Z.-L. Zhang and Z.-Q. Zhang, "An interplay model for rumour spreading and emergency development," Physica A: Statistical Mechanics and its Applications, vol. 388, no. 19, pp. 4159-4166, 2009.

[13] L. Zhao, Q. Wang, J. Cheng et al., “The impact of authorities' media and rumor dissemination on the evolution of emergency," Physica A: Statistical Mechanics and Its Applications, vol. 391, no. 15, pp. 3978-3987, 2012.

[14] L. Huo, P. Huang, and C.-X. Guo, "Analyzing the dynamics of a rumor transmission model with incubation," Discrete Dynamics in Nature and Society, vol. 2012, Article ID 328151, 21 pages, 2012.

[15] X. Zhao and J. Wang, "Dynamical model about rumor spreading with medium," Discrete Dynamics in Nature and Society, vol. 2013, Article ID 586867, 9 pages, 2013.
[16] N. Zhang, H. Huang, B. Su, J. Zhao, and B. Zhang, "Dynamic 8-state ICSAR rumor propagation model considering official rumor refutation," Physica A: Statistical Mechanics and Its Applications, vol. 415, pp. 333-346, 2014.

[17] L. Huo, T. Lin, and P. Huang, "Dynamical behavior of a rumor transmission model with psychological effect in emergency event," Abstract and Applied Analysis, vol. 2013, Article ID 282394, 9 pages, 2013.

[18] H. Hu and L. Huang, "Stability and Hopf bifurcation analysis on a ring of four neurons with delays," Applied Mathematics and Computation, vol. 213, no. 2, pp. 587-599, 2009.

[19] J. Wu, Theory and Applications of Partial Functional Differential Equations, vol. 119, Springer Science \& Business Media, 1996.

[20] W. Zuo, "Global stability and hopf bifurcations of a beddingtondeangelis type predator-prey system with diffusion and delays," Applied Mathematics and Computation, vol. 223, pp. 423-435, 2013. 


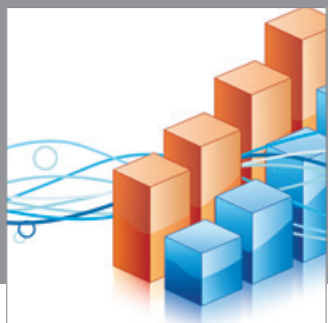

Advances in

Operations Research

mansans

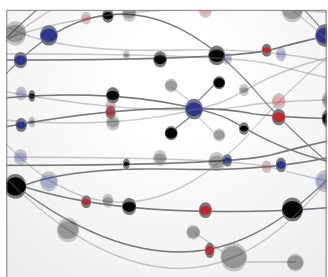

The Scientific World Journal
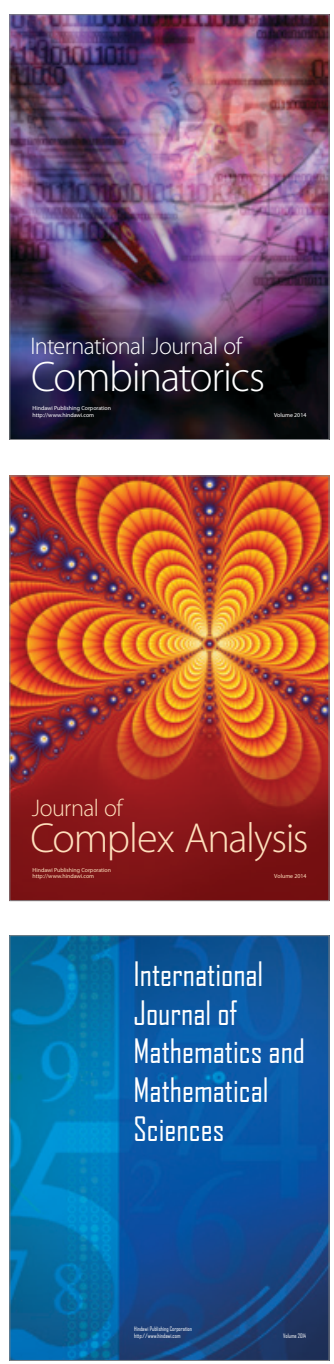
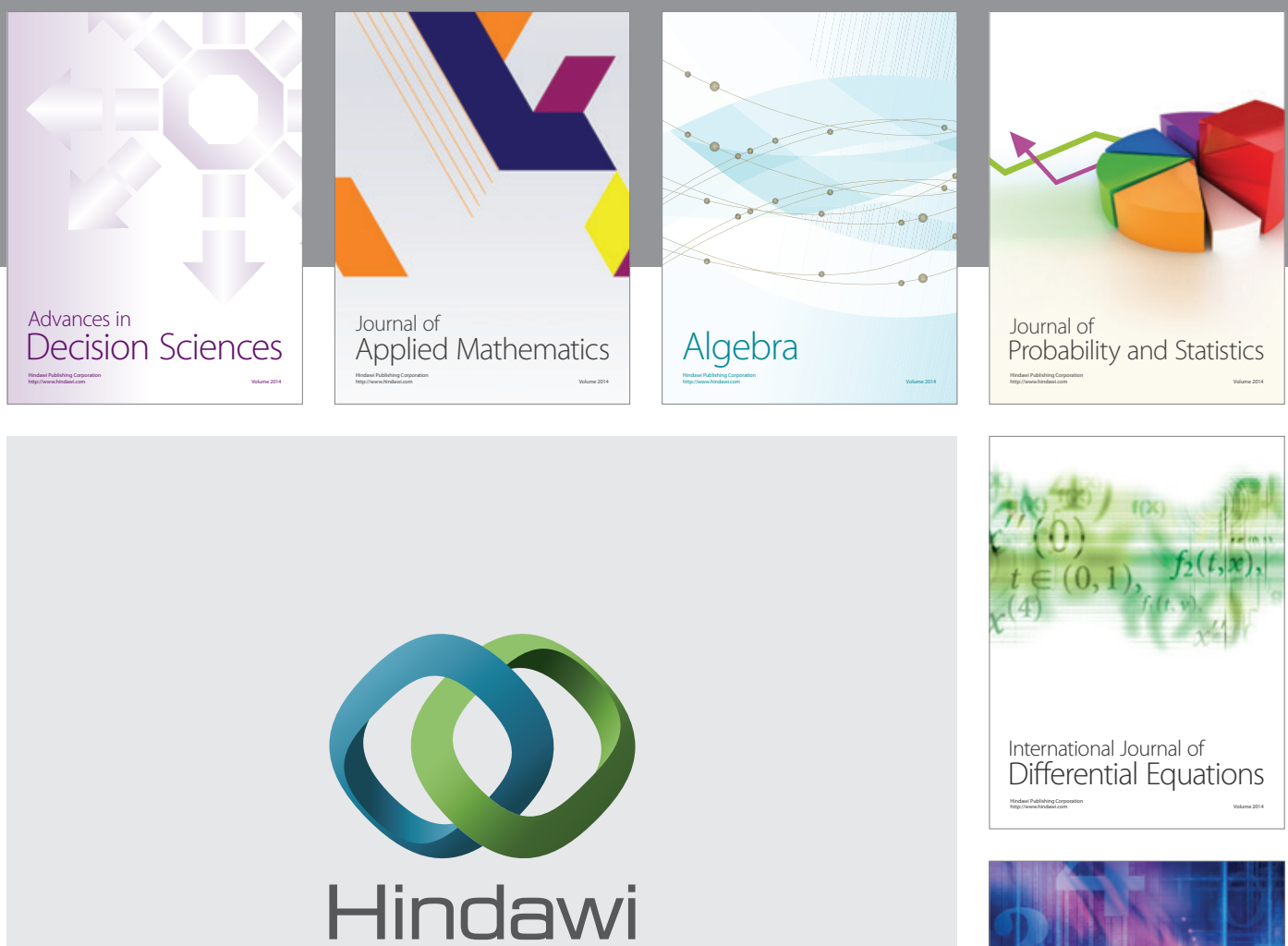

Submit your manuscripts at http://www.hindawi.com
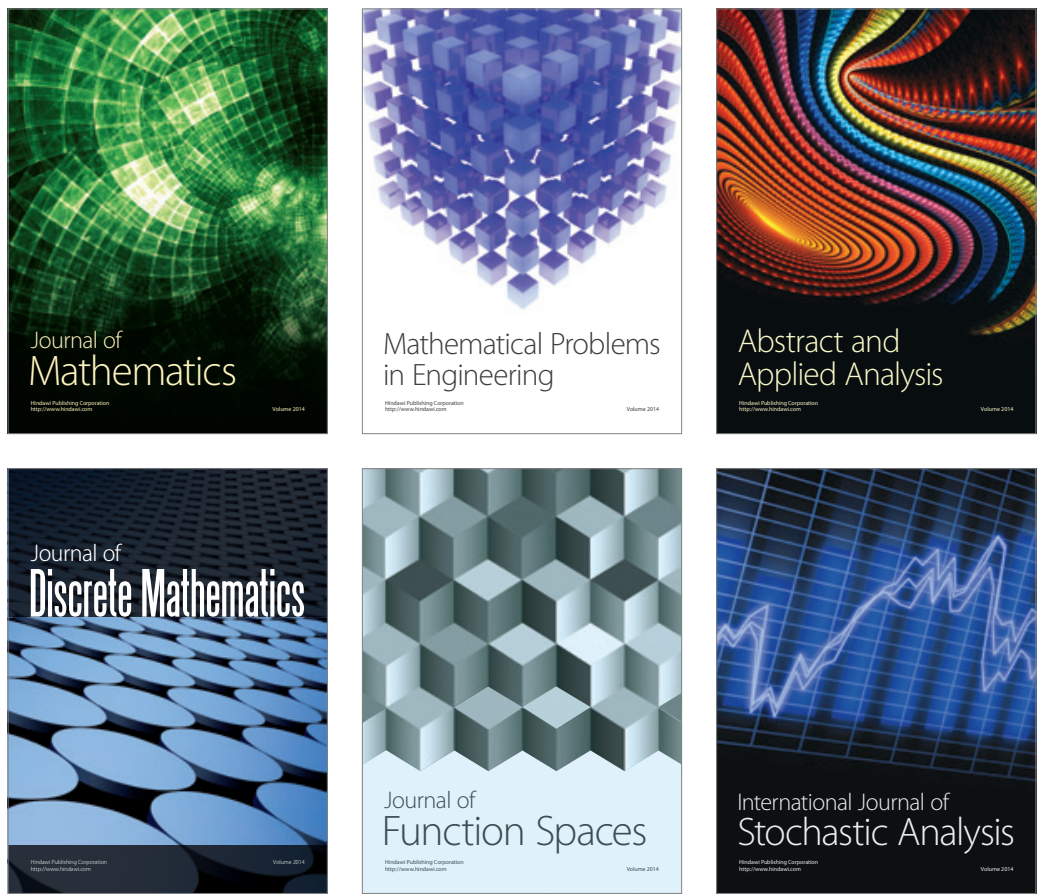

Journal of

Function Spaces

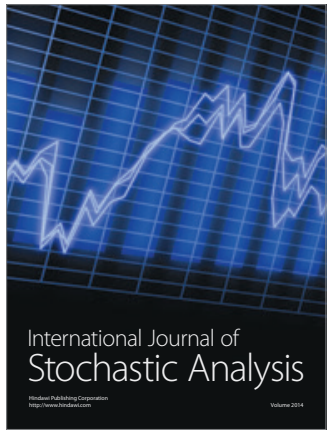

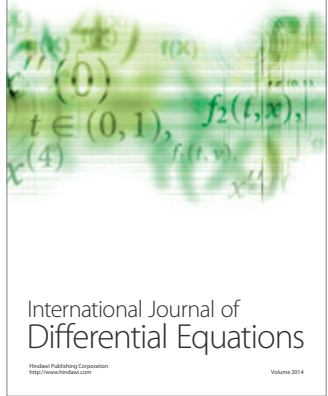
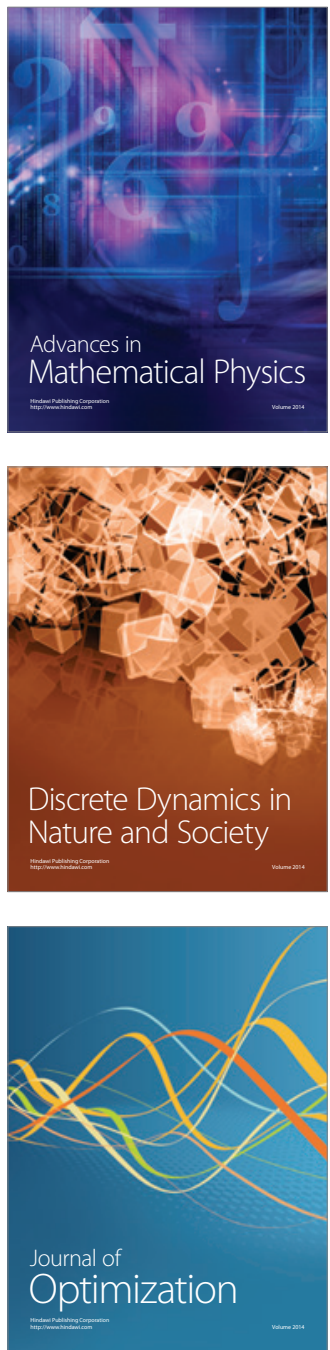